\title{
ESTIMATIVA DA EVAPOTRANSPIRAÇÃO DE REFERÊNCIA (ETo PADRÃO FAO), PARA MINAS GERAIS, NA AUSÊNCIA DE ALGUNS DADOS CLIMÁTICOS
}

Doi:http://dx.doi.org/10.1590/1809-4430-Eng.Agric.v35n 1p 39-50/2015

\section{LEONIDAS P. DE ALENCAR ${ }^{1}$, GILB ERTO C. SEDIYAMA ${ }^{2}$, EVERARDO C. MANTOVANI ${ }^{3}$}

\begin{abstract}
RESUMO: A equação para determinar a evapotranspiração de referência (ETo), método de Penman-Monteith, parametrizada pela FAO (PM-FAO56), requer dados que, muitas vezes, não estão disponíveis na maioria das estações climatológicas. Para superar o problema da disponibilidade de dados climáticos, o boletim 56 da FAO propõe vários procedimentos para estimar a ETo na ausência dos dados de radiação, umidade relativa e velocidade do vento, e posterior substituição. Baseado nisto, o objetivo do presente estudo foi comparar a ETo estimada na ausência de dados de velocidade do vento ou da umidade relativa ou radiação solar com a ETo estimada, com os dados completos para várias localidades de Minas Gerais. A comparação foi realizada utilizando o coeficiente angular da regressão, o coeficiente de determinação, o índice de concordância de Willmott e os erros associados a cada metodologia. Verificou-se que, na ausência de dados de umidade relativa e velocidade do vento, o método de Penman-Monteith apresenta pequenos erros de estimativa. Na ausência dos dados de radiação, os erros são maiores em relação às outras variáveis. $\mathrm{Na}$ disponibilidade apenas das temperaturas máximas e mínimas, o método de PM-FAO56 apresenta desempenho superior ao método de Hargreaves-Samani.
\end{abstract}

PALAVRAS-CHAVE: dados ausentes, evapotranspiração de referência, penman- monteith.

\section{ESTIMATION OF REFERENCE EVAPOTRANSPIRATION (ET $\left.{ }_{0}\right)$ UNDER FAO STANDARDS WITH MISSING CLIMATIC DATA IN MINAS GERAIS, BRAZIL}

\begin{abstract}
The FAO 56 Penman-Monteith (PM) equation for reference evapotranspiration (ETo) estimation requires climatic data that are often not available at most weather stations. To overcome the problem of climate data availability, the FAO-56 report describes several procedures to estimate ETo in the absence of solar radiation, relative humidity and wind speed data with their subsequent replacement. Based on this, this study aimed to compare the ETo estimated with the lack of wind speed or relative humidity or solar radiation data and the estimations using complete data for diverse locations within Minas Gerais State, Brazil. The comparison was performed using coefficient of linear regression and determination, the Willmott's index of agreement and each method errors. As a result, it was found that in the absence of relative humidity and wind speed, the PM equation had small estimation errors. In contrast, for radiation data absence, errors were larger in relation to the other variables. With only maximum and minimum temperatures, the FAO 56-PM method outperformed the Hargreaves-Samani one.
\end{abstract}

KEYWORD: missing data, reference evapotranspiration, penman-monteith method.

\section{INTRODUÇÃO}

Estimativas precisas da evapotranspiração (ET) são essenciais para identificar as variações temporais sobre a necessidade de irrigação, melhorar a alocação dos recursos hídricos e avaliar o efeito do uso da terra e as mudanças na gestão do balanço hídrico (ORTEGA-FARIAS et al., 2009).

\footnotetext{
${ }^{1}$ Eng $^{\circ}$ A grícola e Ambiental, Prof. Doutor, Universidade Federal da Grande Dourado, UFGD/Dourados MS, Fone: (0 xx 67) 3410-2352, lpaufv@ hotmail.com.

${ }^{2}$ Eng $^{\mathbf{0}}$ A grícola, Prof. Doutor, Departamento de Engenharia Agrícola, UFV/Viçosa-MG, g.sediy ama@ufv.br.

${ }^{3}$ Eng $^{\circ}$ A grícola, Prof. Doutor, Departamento de Engenharia Agrícola, UFV/Viçosa-MG, everardo@ufv.br. 
Os principais elementos climáticos que afetam a evapotranspiração são: radiação solar, temperatura do ar, déficit de pressão de vapor e velocidade do vento. O tipo da cultura, a densidade, a variedade e a fase de crescimento também afetam a evapotranspiração, já que diferenças na resistência estomática, altura da cultura, rugosidade do dossel, refletividade e cobertura do solo pela vegetação resultam em diferentes valores de ET sob as mesmas condições climáticas e de solo (ALLEN et al., 1998).

Considerando a grande variedade de modelos, há aqueles que têm uma boa base física e teórica, mas requerem vários parâmetros que não estão sempre disponíveis para uso, como o método de Penman-Monteith. Por outro lado, há aqueles mais simples, exigindo apenas a temperatura do ar (Holdridge) e, adicionalmente, a latitude do lugar (Hargreaves e Samani) (VEGA \& JARA 2009).

O método de Penman-Monteith (FAO) foi escolhido como o método-padrão para estimar ETo, porque se aproxima da evapotranspiração do padrão grama nos locais avaliados e apresenta superioridade em relação aos outros métodos (CAI et al., 2007; GAVILAN et al., 2007; XING et al., 2008). O método é baseado em processos físicos e, explicitamente, incorpora os parâmetros fisiológicos e aerodinâmicos. Existem vários métodos para estimar a evapotranspiração, mas suas performances, em diferentes ambientes, variam, já que a maioria apresenta empirismo em sua concepção (SENTELHAS et al.,2010).

Depois da publicação do boletim 56 da FAO (ALLEN et al., 1998), o método de Penman-Monteith/FAO passou a ser recomendado como o método-padrão para determinar a ETo, mesmo considerando que, em condições climáticas especiais, pode levar a erros próximos a 30\% (WIDMOSER, 2009).

No entanto, em alguns casos, o uso do método de PM FAO é restrito pela falta de algumas variáveis de entrada. Nesse sentido, ALLEN et al. (1998) sugerem procedimentos para estimativa de dados climáticos ausentes, como radiação, umidade relativa e velocidade do vento. Tais procedimentos têm exigido sua avaliação em diferentes países e climas para testar sua viabilidade, como feito por STÖCKLE et al. (2004), para cinco locais na Holanda, Espanha, Filipinas, EUA e Síria, por POPOVA et al. (2006), para a Bulgária, por JABLOUN \& S AHLI (2008), para a Tunísia, e SENTELHAS et al. (2010), para algumas localidades no sul de Ontário, no Canadá.

ALLEN et al. (1998) propõem o uso da equação de Hargreaves (HARGREAVES \& SAMANI, 1985) como alternativa para a estimativa da evapotranspiração, quando somente os dados de temperatura do ar são disponíveis nas estações meteorológicas.

Assim, o objetivo deste estudo foi quantificar o impacto sobre as estimativas da ETo resultantes da utilização de dados limitados, seguindo os procedimentos descritos por ALLEN et al. (1998), para o Estado de Minas Gerais.

\section{MATERIAL E MÉTODOS}

\section{Local e base de dados}

O estudo foi realizado para o Estado de Minas Gerais, situado entre os paralelos $14^{\circ} 13^{\prime} 57^{\prime \prime} \mathrm{e}$ $22^{\circ} 55^{\prime} 22^{\prime \prime}$ de latitude sul e os meridianos de $39^{\circ} 51^{\prime} 23^{\prime \prime}$ e $51^{\circ} 02^{\prime} 45^{\prime \prime}$ de longitude oeste.

Foram utilizadas 20 estações climatológicas convencionais pertencentes à rede nacional de observações meteorológicas de superfície, do Instituto Nacional de Meteorologia (INMET). Para o cálculo da ETo, foram utilizados dados diários referentes a um período de 5 anos, de 2000 a 2005 . Os dias que apresentavam falhas em qualquer variável do modelo foram descartados da análise. 
$\mathrm{Na}$ Tabela 1, estão descritas as localizações das diversas estações climatológicas, que foram utilizadas com suas respectivas coordenadas geográficas.

TABELA 1. Estações meteorológicas do INMET, em Minas Gerais, utilizadas no estudo. INMET's assessed meteorological stations within Minas Gerais State, Brazil.

\begin{tabular}{ccccc}
\hline Código & Cidade & Latitude & Longitude & Altitude (m) \\
\hline 83384 & Arinos & $15^{\circ} 55^{\prime}$ & $46^{\circ} 06^{\prime}$ & 519,0 \\
83514 & Capinópolis & $18^{\circ} 41^{\prime}$ & $49^{\circ} 34^{\prime}$ & 621,5 \\
83574 & Frutal & $20^{\circ} 02^{\prime}$ & $48^{\circ} 56^{\prime}$ & 543,7 \\
83543 & Governador Valadares & $18^{\circ} 51^{\prime}$ & $41^{\circ} 56^{\prime}$ & 170,0 \\
83521 & Ituiutaba & $18^{\circ} 58^{\prime}$ & $49^{\circ} 30^{\prime}$ & 560,0 \\
83395 & Janaúba & $15^{\circ} 47^{\prime}$ & $43^{\circ} 18^{\prime}$ & 516,0 \\
83386 & Januária & $15^{\circ} 27^{\prime}$ & $44^{\circ} 00^{\prime}$ & 473,7 \\
83481 & João Pinheiro & $17^{\circ} 42^{\prime}$ & $46^{\circ} 10^{\prime}$ & 761,3 \\
83389 & Mocambinho & $15^{\circ} 05^{\prime}$ & $44^{\circ} 01^{\prime}$ & 452,0 \\
83388 & Monte Azul & $15^{\circ} 05^{\prime}$ & $42^{\circ} 45^{\prime}$ & 604,7 \\
83437 & Montes Claros & $16^{\circ} 43^{\prime}$ & $43^{\circ} 52^{\prime}$ & 647,2 \\
83479 & Paracatu & $17^{\circ} 13^{\prime}$ & $46^{\circ} 52^{\prime}$ & 712,3 \\
83531 & $18^{\circ} 36^{\prime}$ & $46^{\circ} 31^{\prime}$ & 944,1 \\
83393 & Patos de Minas & $16^{\circ} 00^{\prime}$ & $41^{\circ} 17^{\prime}$ & 649,9 \\
83483 & Pedra Azul & $17^{\circ} 21^{\prime}$ & $44^{\circ} 55^{\prime}$ & 505,4 \\
83441 & Pirapora & $16^{\circ} 10^{\prime}$ & $42^{\circ} 18^{\prime}$ & 471,3 \\
83492 & Salinas & $17^{\circ} 51^{\prime}$ & $41^{\circ} 31^{\prime}$ & 357,3 \\
83577 & Teófilo Otoni & $19^{\circ} 45^{\prime}$ & $47^{\circ} 55^{\prime}$ & 743,9 \\
83428 & Uberaba & $16^{\circ} 22^{\prime}$ & $46^{\circ} 33^{\prime}$ & 460,0 \\
83642 & Unaí & $20^{\circ} 45^{\prime}$ & $42^{\circ} 51^{\prime}$ & 692,9 \\
\hline
\end{tabular}

\section{Método de Hargreaves-Samani}

O método de Hargreaves-Samani [eq.(1)] pode ser uma alternativa para situações em que não se têm dados medidos de radiação solar à superfície, umidade relativa e velocidade do vento:

$$
E T=0,0023\left(T_{\max }-T_{\min }\right)^{0,5}\left(T_{\text {med }}+17,8\right) R a
$$

em que,

$\mathrm{T}_{\text {máx }}$ - temperatura máxima do dia, ${ }^{\circ} \mathrm{C}$;

$\mathrm{T}_{\text {mín }}$ - temperatura mínima do dia, ${ }^{\circ} \mathrm{C}$;

$\mathrm{T}_{\text {méd }}$ - temperatura média do dia, ${ }^{\circ} \mathrm{C}$, e

$\mathrm{Ra}$ - radiação extraterrestre, $\mathrm{mm} \mathrm{d}^{-1}$.

\section{Método de Penman-Monteith FAO}

Os dados meteorológicos diários utilizados para a obtenção da evapotranspiração de referência diária foram: temperatura máxima, temperatura mínima, umidade relativa, velocidade do vento e insolação. Para o cálculo da evapotranspiração e verificação das possíveis ausências de alguns elementos climáticos na evapotranspiração, utilizou-se da equação de Penman-Monteith (FAO) para intervalo de tempo de 24 horas. 


$$
\text { ETo }=\frac{0,408 \Delta\left(\mathrm{R}_{\mathrm{n}}-\mathrm{G}\right)+\gamma \frac{900}{\mathrm{~T}+273} \mathrm{u}_{2}\left(\mathrm{e}_{\mathrm{s}}-\mathrm{e}_{\mathrm{a}}\right)}{\Delta+\gamma\left(1+0,34 \mathrm{u}_{2}\right)}
$$

em que,

ETo - evapotranspiração de referência $\left(\mathrm{mm} \mathrm{d}^{-1}\right)$;

$\mathrm{Rn}$ - saldo de radiação à superfície da cultura $\left(\mathrm{MJ} \mathrm{m}^{-2} \mathrm{~d}^{-1}\right)$;

$\mathrm{G}$ - densidade do fluxo de calor do solo $\left(\mathrm{MJ} \mathrm{m}^{-2} \mathrm{~d}^{-1}\right)$;

$\mathrm{T}$ - temperatura do ar a $2 \mathrm{~m}$ de altura $\left({ }^{\circ} \mathrm{C}\right)$;

$\mathrm{u}_{2}$ - velocidade de vento a $2 \mathrm{~m}$ de altura $\left(\mathrm{m} \mathrm{s}^{-1}\right)$;

$\mathrm{e}_{\mathrm{s}}$ - pressão de vapor de saturação $(\mathrm{kPa})$;

$\mathrm{e}_{\mathrm{a}}$ - pressão parcial de vapor $(\mathrm{kPa})$;

$\Delta$ - declividade da curva de pressão de vapor de saturação $\left(\mathrm{kPa}^{\circ} \mathrm{C}^{-1}\right)$, e

$\gamma$ - coeficiente psicrométrico $\left(\mathrm{kPa}^{\circ} \mathrm{C}^{-1}\right)$.

As equações padronizadas para o cálculo de todos os parâmetros da [eq. (2)] são apresentadas em ALLEN et al. (1998).

\section{Estimativa dos dados ausentes}

O boletim N ${ }^{\circ} 56$ da FAO (FAO56) apresenta a metodologia que se deve utilizar na ausência de dados de velocidade do vento, radiação solar e umidade relativa. Quando apenas os registros de temperatura estão disponíveis, dois procedimentos são propostos por ALLEN et al. (1998). O primeiro deles é o método de Penman-Monteith (FAO 56) com dados estimados de velocidade do vento, umidade relativa e radiação. O segundo procedimento é o método de Hargreaves-Samani.

\section{Umidade Relativa}

Na ausência de dados de umidade relativa ou quando os dados são de qualidade duvidosa, o déficit de pressão de vapor $\left(\mathrm{e}_{\mathrm{s}}-\mathrm{e}_{\mathrm{a}}\right)$ pode ser estimado com base em dados de temperatura. Uma estimativa da pressão parcial de vapor $\left(\mathrm{e}_{\mathrm{a}}\right)$ pode ser obtida, assumindo-se que a temperatura do ponto de orvalho $\left(\mathrm{T}_{\mathrm{po}}\right)$ é próxima da temperatura mínima diária $\left(\mathrm{T}_{\mathrm{mín}}\right)$. Esta afirmação pressupõe implicitamente que, ao amanhecer, quando a temperatura está próxima da $\mathrm{T}_{\text {mín }} \mathrm{o}$ ar está praticamente saturado, isto é, a umidade relativa do ar é quase $100 \%$.

Se a $\mathrm{T}_{\text {mín }}$ é usada para representar $\mathrm{T}_{\text {po }}$ então:

$$
e_{a}=e\left(T_{\min }\right)=0,611 \exp \left[\frac{17,27 \times T_{\min }}{T_{\min }+237,3}\right]
$$

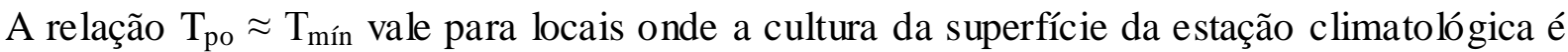
adequadamente irrigada. Contudo, particularmente em regiões áridas, o ar pode não estar saturado, quando a temperatura está em seu mínimo; assim, a $\mathrm{T}_{\text {mín }}$ pode ser maior do que a $\mathrm{T}_{\mathrm{po}}$. Nessa condição, uma calibração adicional pode ser necessária para estimar as temperaturas do ponto de orvalho (ALLEN et al., 1998). Para as localidades de Janaúba, Januária, Mocambinho, Montes Claros, Salinas e Teófilo Otoni, foi subtraído $1^{\circ} \mathrm{C}$ da $\mathrm{T}_{\min } \mathrm{e}$, para a localidade de Monte Azul, foram subtraídos $2^{\circ} \mathrm{C}$. 


\section{Velocidade do ve nto}

Como a variação da velocidade média do vento em períodos mensais é relativamente baixa, os valores mensais da velocidade do vento podem ser estimados para o cálculo da ETo. As estimativas da média da velocidade do vento podem ser obtidas a partir de informações disponíveis para o clima regional, mas devem-se levar em conta as alterações sazonais (ALLEN et al., 1998).

Quando os dados de vento na região não estão disponíveis, um valor de $2 \mathrm{~m} \mathrm{~s}^{-1}$ pode ser usado como uma estimativa provisória. Este valor é uma média de mais de 2.000 estações meteorológicas estudadas ao redor do mundo. No presente estudo, foi adotado o valor de $2 \mathrm{~m} \mathrm{~s}^{-1}$ para todas as estações utilizadas.

\section{Radiação solar}

A diferença entre as temperaturas máxima e mínima está relacionada com o grau de cobertura de nuvens em um determinado local. Desta maneira, para o cálculo da radiação solar foi utilizada a Equação 4, proposta por HARGREAVES \& SAMANI (1985), que estima a radiação solar global pela diferença das temperaturas máximas e mínimas diárias do ar. A forma ajustada e validada para várias estações em diferentes condições climáticas é:

$$
R_{s}=k_{r} \sqrt{\left(T_{\max }-T_{\min }\right)} R_{a}
$$

em que,

Ra é a radiação extraterrestre $\left(\mathrm{MJ} \mathrm{m}^{-2} \mathrm{~d}^{-1}\right), \mathrm{e}$

$\mathrm{k}_{\mathrm{r}}$, o coeficiente de ajuste $\left({ }^{\circ} \mathrm{C}^{-0,5}\right)$.

O coeficiente de ajuste varia de 0,16 a 0,19 , sendo que o valor de 0,16 é utilizado para as regiões de interior e 0,19 para as regiões costeiras. No estudo, foi utilizado o valor de 0,16.

\section{Avaliação das metodologias}

A análise de desempenho para as diferentes metodologias foi feita comparando os valores de ETo obtidos na ausência dos dados de velocidade do vento, radiação e umidade relativa, individualmente, com os valores obtidos utilizando os dados completos. Comparou-se, também, a ETo obtida com a ausência de dados das três variáveis simultaneamente. Por fim comparou-se o método de Hargreaves-Samani para a determinação da ETo, com o método-padrão. Na comparação dos dados, mantiveram-se os valores originais das outras variáveis.

O desempenho dos métodos para cada localidade foi avaliado pela análise de regressão, com o coeficiente linear passando pela origem $(\mathrm{a}=0)$, porque, numa situação ideal, a ETo é zero para outras equações, quando não há evapotranspiração. O coeficiente angular (b) foi utilizado como uma medida de exatidão, enquanto o coeficiente de determinação $\left(\mathrm{r}^{2}\right)$ foi considerado como uma medida de precisão. Um método ideal deve apresentar valores de $b=1$ e $r^{2}=1$.

Os valores da ETo obtidos pelo método de PM-FAO56 e os computados na ausência de algum dado foram comparados usando uma série de estatísticas propostas por WILLMOTT (1982), para analisar o erro associado a cada método.

$\mathrm{O}$ viés médio dos modelos foi calculado pela equação:

$$
\mathrm{VM}=\mathrm{N}^{-1} \sum_{\mathrm{i}=1}^{\mathrm{N}}\left(\mathrm{P}_{\mathrm{i}}-\mathrm{O}_{\mathrm{i}}\right)
$$

em que,

N é o número de observações;

Pi são valores estimados da ETo $\left(\mathrm{mm} \mathrm{d}^{-1}\right)$ com dados ausentes, e

Oi são valores de ETo $\left(\mathrm{mm} \mathrm{d}^{-1}\right)$ calculados com os dados completos. 
Os erros de cada metodologia foram calculados pela raiz do erro quadrático médio (REQM) e pelo erro absoluto médio (EAM), dados pelas seguintes equações:

$$
\begin{aligned}
& \text { REQM }=\sqrt{\left(\mathrm{N}^{-1} \sum_{\mathrm{i}=1}^{\mathrm{N}}\left(\mathrm{P}_{\mathrm{i}}-\mathrm{O}_{\mathrm{i}}\right)^{2}\right)} \\
& \text { EAM }=\mathrm{N}^{-1} \sum_{\mathrm{i}=1}^{\mathrm{N}}\left|\mathrm{P}_{\mathrm{i}}-\mathrm{O}_{\mathrm{i}}\right|
\end{aligned}
$$

A aproximação dos valores de ETo, estimados com os dados completos, em comparação aos valores obtidos na ausência das variáveis, foi avaliada pelo índice de concordância (d) (WILLMOTT, 1982).

$$
\mathrm{d}=1-\left[\frac{\sum_{\mathrm{i}=1}^{\mathrm{N}}\left(\mathrm{P}_{\mathrm{i}}-\mathrm{O}_{\mathrm{i}}\right)^{2}}{\sum_{\mathrm{i}=1}^{\mathrm{N}}\left(\left|\mathrm{P}_{\mathrm{i}}-\overline{\mathrm{O}}\right|-\left|\mathrm{O}_{\mathrm{i}}-\overline{\mathrm{O}}\right|\right)^{2}}\right], \quad 0 \leq \mathrm{d} \leq 1
$$

em que,

$\overline{\mathrm{O}}$ é a média dos valores de ETo obtida com os dados completos (mm.d $\left.\mathrm{d}^{-1}\right)$.

Esta medida condensa todas as diferenças entre as estimativas do modelo e as observações em uma estatística quantitativa. $\mathrm{O}$ índice de concordância é uma medida de quão bem o modelo estima a proximidade dos dados à média observada. $\mathrm{O}$ índice de concordância tem um intervalo variando entre $-\infty$ e 1 , sendo que valores próximos a 1 mostram concordância adequada.

\section{RESULTADOS E DISCUSSÃO}

\section{Velocidade do ve nto}

Na Tabela 2, encontram-se os valores do coeficiente angular da equação de regressão (b), coeficiente de determinação $\left(\mathrm{r}^{2}\right)$ e os índices estatísticos utilizados para a relação entre a ETo estimada pelo método-padrão de Penman-Monteith (dados completos), com a ETo estimada na ausência dos dados de velocidade do vento.

$\mathrm{Na}$ ausência de dados de velocidade do vento, o método de Penman-Monteith mostra-se como boa alternativa para a obtenção da ETo para a quase totalidade das localidades de Minas Gerais.

As cidades de Janaúba e Monte Azul apresentaram os menores valores de $\mathrm{r}^{2}$, que foram de 0,217 e 0,309, respectivamente. Para essas localidades, também foram encontrados os menores valores do índice de concordância de Willmott.

O viés médio encontrado mostra uma superestimativa da ETo para a maioria das localidades, exceto para Monte Azul e Montes Claros. O VM variou de - 0,28 a 0,45 $\mathrm{mm} \mathrm{d}^{-1}$, com uma média de $0,14 \mathrm{~mm} \mathrm{~d}^{-1}$, mostrando que, na ausência dos dados de velocidade do vento, o método de Penman-Monteith tende a superestimar a ETo obtida com os dados completos em 0,14 $\mathrm{mm} \mathrm{d}^{-1}$, em média, para todas as localidades.

As superestimativas da ETo ocorrem por causa de a velocidade média do vento da localidade ser inferior ao valor de $2 \mathrm{~m} \mathrm{~s}^{-1}$, que é utilizado na metodologia. 
TABELA 2. Coeficiente angular (b), coeficiente de determinação $\left(r^{2}\right)$ e índices estatísticos para as relações entre a ETo estimada pelo método de Penman-Monteith com os dados completos, e ETo estimada na ausência dos dados de velocidade do vento para diferentes localidades. Linear regression coefficient (b), coefficient of determination $\left(r^{2}\right)$ and statistical indices for the relationship between ETo estimated by the Penman-Monteith with complete data and ETo estimated in the absence of wind speed data at diffe rent locations.

\begin{tabular}{ccccccc}
\hline Cidades & $\mathbf{b}$ & $\mathbf{r}^{2}$ & $\mathbf{V M}$ & REQM & EAM & $\mathbf{d}$ \\
\hline Arinos & 1,097 & 0,858 & 0,41 & 0,59 & 0,46 & 0,93 \\
Capinópolis & 0,991 & 0,721 & 0,03 & 0,58 & 0,34 & 0,93 \\
Frutal & 1,111 & 0,888 & 0,45 & 0,58 & 0,46 & 0,94 \\
Gov.Valadares & 1,092 & 0,751 & 0,38 & 0,68 & 0,51 & 0,92 \\
Ituiutaba & 1,019 & 0,856 & 0,11 & 0,40 & 0,28 & 0,96 \\
Janaúba & 1,011 & 0,217 & 0,19 & 0,92 & 0,75 & 0,81 \\
Januária & 1,000 & 0,789 & 0,08 & 0,48 & 0,38 & 0,95 \\
João Pinheiro & 1,037 & 0,869 & 0,18 & 0,42 & 0,33 & 0,96 \\
Mocambinho & 1,035 & 0,902 & 0,16 & 0,35 & 0,27 & 0,97 \\
Monte Azul & 0,910 & 0,309 & $-0,28$ & 0,95 & 0,73 & 0,83 \\
Montes Claros & 0,986 & 0,938 & $-0,03$ & 0,28 & 0,19 & 0,98 \\
Paracatu & 0,992 & 0,796 & 0,04 & 0,46 & 0,35 & 0,96 \\
Patos de Minas & 1,025 & 0,850 & 0,14 & 0,42 & 0,29 & 0,96 \\
Pedra Azul & 1,075 & 0,945 & 0,30 & 0,39 & 0,33 & 0,97 \\
Pirapora & 1,009 & 0,929 & 0,07 & 0,31 & 0,22 & 0,98 \\
Salinas & 1,058 & 0,824 & 0,30 & 0,49 & 0,43 & 0,95 \\
Teófilo Otoni & 1,056 & 0,977 & 0,21 & 0,28 & 0,23 & 0,98 \\
Uberaba & 1,002 & 0,820 & 0,06 & 0,47 & 0,33 & 0,95 \\
Unaí & 1,081 & 0,850 & 0,35 & 0,53 & 0,41 & 0,94 \\
Viçosa & 1,021 & 0,986 & 0,08 & 0,14 & 0,11 & 0,99 \\
\hline
\end{tabular}

VM - viés médio, REQM - raiz do erro quadrático médio, EAM - erro absoluto médio, d - índice de concordância de Willmott

O valor da REQM variou de 0,14 a $0,95 \mathrm{~mm} \mathrm{~d}^{-1}$, sendo que os maiores erros foram encontrados para as cidades de Janaúba e Monte Azul.

O EAM variou de 0,11 a $0,75 \mathrm{~mm} \mathrm{~d}^{-1}$. Nota-se que, para a cidade de Arinos, apesar de ter tido o maior viés para a velocidade do vento, essa localidade apresentou um EAM de $0,46 \mathrm{~mm} \mathrm{~d}^{-1}$. O EAM médio para as localidades foi de $0,36 \mathrm{~mm} \mathrm{~d}^{-1}$.

Apesar dos bons resultados obtidos na ausência dos dados de velocidade do vento, espera-se que melhores resultados possam ser obtidos utilizando os valores médios anuais ou mensais de cada estação, melhorando-se assim suas estimativas (POPOVA et al., 2006). 


\section{Umidade Relativa}

Na Tabela 3, encontram-se os valores das análises estatísticas realizadas para o conjunto de dados de ETo, estimada na ausência de dados de umidade relativa.

TABELA 3. Coeficiente angular (b), coeficiente de determinação $\left(r^{2}\right)$ e índices estatísticos para as relações entre a ETo estimada pelo método de Penman-Monteith com os dados completos e ETo estimada na ausência dos dados de umidade relativa para diferentes localidades. Linear regression coefficient $(b)$, coefficient of determination $\left(r^{2}\right)$ and statistical indices for the relationship between ETo estimated by the PenmanMonteith with complete data and ETo estimated in the absence of data relative humidity at different locations.

\begin{tabular}{ccccccc}
\hline Cidades & $\mathbf{b}$ & $\mathbf{r}^{\mathbf{2}}$ & $\mathbf{V M}$ & REQM & EAM & d \\
\hline Arinos & 0,995 & 0,989 & 0,01 & 0,11 & 0,04 & 0,99 \\
Capinópolis & 0,987 & 0,928 & $-0,01$ & 0,30 & 0,18 & 0,98 \\
Frutal & 0,995 & 0,984 & $-0,01$ & 0,14 & 0,08 & 0,99 \\
Gov.Valadares & 0,994 & 0,991 & $-0,02$ & 0,11 & 0,06 & 0,99 \\
Ituiutaba & 1,005 & 0,946 & 0,04 & 0,24 & 0,16 & 0,98 \\
Janaúba & 0,960 & 0,918 & $-0,14$ & 0,36 & 0,22 & 0,97 \\
Januária & 1,015 & 0,940 & 0,11 & 0,28 & 0,22 & 0,98 \\
João Pinheiro & 0,987 & 0,965 & $-0,04$ & 0,20 & 0,13 & 0,99 \\
Mocambinho & 1,001 & 0,962 & 0,02 & 0,19 & 0,12 & 0,99 \\
Monte Azul & 0,947 & 0,912 & $-0,20$ & 0,44 & 0,29 & 0,97 \\
Montes Claros & 0,992 & 0,943 & 0,01 & 0,26 & 0,19 & 0,99 \\
Paracatu & 0,996 & 0,931 & 0,02 & 0,29 & 0,19 & 0,98 \\
Patos de Minas & 0,988 & 0,957 & $-0,01$ & 0,22 & 0,13 & 0,99 \\
Pedra Azul & 1,000 & 0,994 & 0,02 & 0,10 & 0,15 & 0,99 \\
Pirapora & 0,988 & 0,955 & $-0,01$ & 0,24 & 0,15 & 0,99 \\
Salinas & 1,032 & 0,953 & 0,15 & 0,27 & 0,21 & 0,98 \\
Teófilo Otoni & 1,015 & 0,988 & 0,07 & 0,13 & 0,10 & 0,99 \\
Uberaba & 0,994 & 0,933 & 0,01 & 0,29 & 0,19 & 0,98 \\
Unaí & 0,985 & 0,962 & $-0,04$ & 0,20 & 0,12 & 0,99 \\
Viçosa & 1,033 & 0,983 & 0,11 & 0,18 & 0,14 & 0,99 \\
\hline
\end{tabular}

VM - viés médio; REQM - raiz do erro quadrático médio; EAM - erro absoluto médio, d - índice de concordân cia de Willmott

$\mathrm{Na}$ ausência de dados de umidade relativa, a metodologia para a substituição dos dados ausentes apresenta seu melhor desempenho. A obtenção da pressão parcial de vapor $\left(\mathrm{e}_{\mathrm{a}}\right)$, a partir dos dados de temperatura mínima, mostrou-se aceitável.

O coeficiente angular das equações de regressão apresenta valores variando de 0,947 a 1,033, próximo do valor ideal. Os valores do coeficiente de determinação mostram que a ETo obtida na ausência dos dados de umidade relativa explica bem a ETo obtida com todos os dados necessários. Verifica-se também que o índice de concordância de Willmott apresentou valores próximos a 1, mostrando uma boa concordância.

$\mathrm{O}$ viés médio $(\mathrm{VM})$, para ausência dos dados de umidade relativa, indica que a metodologia utilizada representou subestimativa da ETo em nove localidades, e a superestimativa da ETo nas outras. A menor subestimativa foi de $0,20 \mathrm{~mm} \mathrm{~d}^{-1}$, para a localidade de Monte Azul, e a maior superestimativa foi de $0,15 \mathrm{~mm} \mathrm{~d}^{-1}$, para a cidade de Salinas.

A REQM variou de 0,11 a $0,44 \mathrm{~mm} \mathrm{~d}^{-1}$, sendo que, novamente, os maiores erros foram encontrados para as cidades de Monte Azul e Janaúba. As outras localidades apresentam valores próximos da REQM.

Assim como o viés médio e a REQM, o EAM apresentou os menores valores. O EAM variou de 0,04 a $0,29 \mathrm{~mm} \mathrm{~d}^{-1}$, com média de $0,15 \mathrm{~mm} \mathrm{~d}^{-1}$, mostrando que, na ausência dos dados de umidade relativa, o método pode ser usado sem grandes erros.

SENTELHAS et al. (2010) verificaram que o método de Penman-Monteith, na ausência de dados de umidade relativa, apresentou os melhores resultados para as localidades estudadas. Este 
mesmo comportamento foi observado, para algumas localidades da Tunísia, por JABLOUN \& SAHLI (2008), que obtiveram os menores valores de REQM na ausência de dados de umidade relativa.

\section{Radiação}

$\mathrm{Na}$ Tabela 4, encontram-se os valores das análises estatísticas realizadas para o conjunto de dados de ETo, estimada na ausência de dados de radiação.

TABELA 4. Coeficiente angular (b), coeficiente de determinação $\left(r^{2}\right)$ e índices estatísticos para as relações entre a ETo estimada pelo método de Penman-Monteith com os dados completos, e ETo estimada na ausência dos dados de radiação para diferentes localidades. Linear regression coefficient (b), coefficient of determination $\left(r^{2}\right)$ and statistical indices for the relationship between ETo estimated by the PenmanMonteith with complete data and ETo estimated in the absence of radiation data at diffe rent locations.

\begin{tabular}{ccccccc}
\hline Cidades & $\mathbf{b}$ & $\mathbf{r}^{2}$ & $\mathbf{V M}$ & REQM & EAM & $\mathbf{d}$ \\
\hline Arinos & 1,008 & 0,647 & 0,11 & 0,54 & 0,41 & 0,92 \\
Capinópolis & 0,947 & 0,570 & $-0,11$ & 0,66 & 0,54 & 0,90 \\
Frutal & 0,977 & 0,697 & 0,05 & 0,54 & 0,42 & 0,93 \\
Gov.Valadares & 0,984 & 0,589 & 0,06 & 0,59 & 0,46 & 0,91 \\
Ituiutaba & 1,042 & 0,795 & 0,22 & 0,49 & 0,36 & 0,94 \\
Janaúba & 0,975 & 0,709 & $-0,03$ & 0,57 & 0,44 & 0,93 \\
Januária & 0,988 & 0,658 & 0,05 & 0,55 & 0,42 & 0,93 \\
João Pinheiro & 0,890 & 0,557 & $-0,36$ & 0,70 & 0,61 & 0,86 \\
Mocambinho & 0,961 & 0,617 & $-0,10$ & 0,51 & 0,39 & 0,91 \\
Monte Azul & 0,882 & 0,613 & $-0,45$ & 0,83 & 0,69 & 0,88 \\
Montes Claros & 0,965 & 0,651 & $-0,04$ & 0,56 & 0,43 & 0,93 \\
Paracatu & 0,966 & 0,596 & $-0,03$ & 0,60 & 0,48 & 0,92 \\
Patos de Minas & 0,992 & 0,579 & 0,08 & 0,59 & 0,46 & 0,91 \\
Pedra Azul & 0,953 & 0,685 & $-0,06$ & 0,55 & 0,43 & 0,93 \\
Pirapora & 0,970 & 0,631 & $-0,01$ & 0,57 & 0,43 & 0,93 \\
Salinas & 1,059 & 0,467 & 0,36 & 0,67 & 0,55 & 0,88 \\
Teófilo Otoni & 1,030 & 0,750 & 0,20 & 0,53 & 0,41 & 0,94 \\
Uberaba & 0,990 & 0,595 & 0,07 & 0,63 & 0,49 & 0,91 \\
Unaí & 1,019 & 0,547 & 0,17 & 0,61 & 0,44 & 0,90 \\
Viçosa & 1,046 & 0,742 & 0,23 & 0,53 & 0,41 & 0,93 \\
\hline
\end{tabular}

VM - viés médio; REQM - raiz do erro quadrático médio; EAM - erro absoluto médio, e d - índ ice de concordância de Willmott.

A ETo, estimada na ausência de dados de radiação solar, apresenta um desempenho aquém, quando comparado com os dados obtidos na ausência de velocidade do vento e umidade relativa. Os resultados mostram um aumento da dispersão dos dados apresentando valores de $\mathrm{r}^{2}$ variando de 0,467 a 0,795 e o índ ice de concordância variando entre 0,86 e 0,94 .

Apesar do aumento da dispersão dos dados e a menor concordância entres os dados, as estimativas da ETo, na ausência dos dados de radiação, foram aceitáveis. O viés médio (VM), na ausência dos dados de radiação, variou de $-0,45$ a $0,36 \mathrm{~mm} \mathrm{~d}^{-1}$. A menor subestimativa foi encontrada para Monte Azul, e a maior superestimativa foi observada em Salinas.

Os valores da REQM variaram entre 0,49 e $0,83 \mathrm{~mm} \mathrm{~d}^{-1}$, apresentando um erro médio de 0,59 $\mathrm{mm} \mathrm{d}^{-1}$, sendo que SENTELHAS et al. (2010) encontraram valores médio de REQM de $0,8 \mathrm{~mm} \mathrm{~d}^{-1}$ para as localidades no sul de Ontário, no Canadá. O EAM variou de 0,36 a 0,69 $\mathrm{mm} \mathrm{d}^{-1}$ com um EAM médio de $0,46 \mathrm{~mm} \mathrm{~d}^{-1}$.

POPOVA et al. (2006) e SENTELHAS et al. (2010) encontraram as piores estimativas (menor concordância) de ETo, na ausência dos dados de radiação, para o sul da Bulgária e para algumas localidades do Canadá, respectivamente. SENTELHAS et al. (2010) relatam que o método, recomendado por ALLEN et al. (1998), para a obtenção da radiação, não é recomendado para as 
localidades estudadas. Os maiores erros associados à ausência dos dados de radiação, estão ligados à equação de Hargreaves e Samani para a estimativa da radiação. Para verificar o método que melhor se ajusta aos dados de radiação, observados de cada localidade, é necessário verificar qual método melhor se adapta às condições climáticas de cada local.

Para Tunísia, JABLOUN \& SAHLI (2008) ajustaram o coeficiente $\mathrm{k}_{\mathrm{r}}$ para cada localidade estudada, melhorando assim a performance do método de Penman-Monteith na ausência dos dados de radiação.

\section{Método de Hargreaves-Samani}

$\mathrm{Na}$ Tabela 5, encontram-se os valores das análises estatísticas realizadas para o conjunto de dados de ETo, estimada na ausência de dados de velocidade do vento, umidade relativa e radiação, utilizando-se do método de Hargreaves-Samani.

TABELA 5. Coeficiente angular (b), coeficiente de determinação $\left(\mathrm{r}^{2}\right)$ e índices estatísticos para as relações entre a ETo estimada pelo método de Penman-Monteith, com os dados completos, e ETo estimada na ausência dos dados de radiação para diferentes localidades. Linear regression coefficient $(b)$, coefficient of determination $\left(r^{2}\right)$ and statistical indices for the relationship between ETo estimated by the PenmanMonteith with complete data and ETo estimated in the absence of radiation data at diffe rent locations.

\begin{tabular}{|c|c|c|c|c|c|c|c|c|c|c|c|c|}
\hline \multirow{2}{*}{$\begin{array}{c}\text { Dado aus ente } \\
\text { Cidades }\end{array}$} & \multicolumn{6}{|c|}{ Velocidade do vento, Umi dade e Radi ação } & \multicolumn{6}{|c|}{ Hargreaves-Samani } \\
\hline & b & $\mathbf{r}^{2}$ & VM & REQM & EAM & d & b & $\mathbf{r}^{2}$ & VM & REQM & EAM & d \\
\hline Arinos & 1,108 & 0,315 & 0,56 & 0,87 & 0,72 & 0,82 & 1,276 & 0,365 & 1,23 & 1,41 & 1,26 & 0,69 \\
\hline Capinópolis & 0,960 & 0,287 & $-0,02$ & 0,76 & 0,59 & 0,85 & 1,107 & 0,319 & 0,59 & 0,98 & 0,79 & 0,81 \\
\hline Frutal & 1,076 & 0,569 & 0,40 & 0,70 & 0,56 & 0,89 & 1,242 & 0,608 & 1,02 & 1,19 & 1,04 & 0,78 \\
\hline Gov.Valadares & 1,097 & 0,385 & 0,50 & 0,81 & 0,67 & 0,85 & 1,280 & 0,446 & 1,13 & 1,30 & 1,16 & 0,75 \\
\hline Ituiutaba & 1,088 & 0,398 & 0,47 & 0,80 & 0,66 & 0,84 & 1,251 & 0,436 & 1,12 & 1,32 & 1,17 & 0,72 \\
\hline Janaúba & 0,980 & 0,450 & 0,13 & 1,18 & 0,97 & 0,65 & 1,108 & 0,330 & 0,70 & 1,44 & 1,21 & 0,62 \\
\hline Januária & 0,972 & 0,302 & 0,02 & 0,71 & 0,56 & 0,87 & 1,098 & 0,389 & 0,58 & 0,92 & 0,74 & 0,84 \\
\hline João Pinheiro & 0,944 & 0,431 & $-0,12$ & 0,66 & 0,54 & 0,87 & 1,088 & 0,458 & 0,46 & 0,82 & 0,63 & 0,84 \\
\hline Mocambinho & 1,000 & 0,453 & 0,09 & 0,59 & 0,47 & 0,88 & 1,127 & 0,520 & 0,64 & 0,88 & 0,72 & 0,81 \\
\hline Monte Azul & 0,841 & 0,470 & $-0,55$ & 1,29 & 1,04 & 0,65 & 0,929 & 0,250 & $-0,12$ & 1,20 & 0,99 & 0,71 \\
\hline Montes Claros & 0,962 & 0,401 & $-0,03$ & 0,68 & 0,53 & 0,88 & 1,082 & 0,487 & 0,47 & 0,82 & 0,66 & 0,86 \\
\hline Paracatu & 0,962 & 0,323 & $-0,02$ & 0,71 & 0,57 & 0,87 & 1,108 & 0,359 & 0,59 & 0,93 & 0,76 & 0,83 \\
\hline Patos de Minas & 1,035 & 0,164 & 0,29 & 0,78 & 0,64 & 0,83 & 1,183 & 0,218 & 0,84 & 1,13 & 0,96 & 0,75 \\
\hline Pedra Azul & 1,030 & 0,579 & 0,23 & 0,63 & 0,50 & 0,91 & 1,188 & 0,610 & 0,80 & 0,99 & 0,83 & 0,83 \\
\hline Pirapora & 0,997 & 0,298 & 0,14 & 0,72 & 0,58 & 0,87 & 1,150 & 0,370 & 0,79 & 1,04 & 0,90 & 0,81 \\
\hline Salinas & 1,084 & 0,100 & 0,50 & 0,84 & 0,69 & 0,82 & 1,121 & 0,172 & 1,05 & 1,23 & 1,10 & 0,73 \\
\hline Teófilo Otoni & 1,140 & 0,569 & 0,63 & 0,83 & 0,71 & 0,86 & 1,292 & 0,642 & 1,15 & 1,28 & 1,16 & 0,78 \\
\hline Uberaba & 1,002 & 0,196 & 0,16 & 0,82 & 0,67 & 0,84 & 1,148 & 0,240 & 0,76 & 1,14 & 0,95 & 0,77 \\
\hline Unaí & 1,101 & 0,162 & 0,54 & 0,88 & 0,71 & 0,80 & 1,268 & 0,225 & 1,19 & 1,39 & 1,23 & 0,68 \\
\hline Viçosa & 1,109 & 0,643 & 0,47 & 0,68 & 0,57 & 0,89 & 1,271 & 0,673 & 0,98 & 1,10 & 0,99 & 0,80 \\
\hline
\end{tabular}

O método de Hargreaves-Samani é bastante utilizado por ser um dos métodos mais simples para a estimativa da evapotranspiração. O método de Hargreaves-Samani requer apenas os dados de temperaturas mínimas e máximas. Comparou-se, assim, a ETo obtida, na ausência dos dados de velocidade do vento, umidade relativa e radiação, e a ET obtida pelo método de HargreavesSamani, com o método de PM-FAO56 na presença de todos os dados.

Pela análise dos coeficientes da regressão, nota-se uma piora dos índices estatísticos na ausência de dados, para o método de Hargreaves-Samani. O coeficiente de determinação para o método de PM-FAO56, na ausência de dados, variou de 0,100 a 0,643, sendo que, para o método de Hargreaves-Samani, o coeficiente variou de 0,172 a 0,673, isto é, grande dispersão dos dados quando comparados com a ETo obtida na presença de todos os dados. 
O índice de concordância de Willmott, para o método de PM-FAO56, foi superior ao encontrado pelo método de Hargreaves-Samani em todas as localidades, exceto para a localidade de Monte Azul.

$\mathrm{Na}$ ausência dos três elementos climáticos estudados, o método de Penman-Monteith tende a superestimar a ETo em 15 das 20 localidades, sendo que, em média, a superestimativa para essas localidades foi de $0,34 \mathrm{~mm} \mathrm{~d}^{-1}$, e a maior superestimativa foi encontrada para a cidade de Teó filo Otoni. Dentre as localidades que apresentaram subestimativa, a cidade de Monte Azul teve o menor viés $\left(-0,55 \mathrm{~mm} \mathrm{~d}^{-1}\right)$. O método de Hargreaves-Samani apresentou maiores erros quando comparado com o método de PM-FAO56, apresentando uma superestimativa da evapotranspiração em todas as localidades, exceto para Monte Azul. Em média, o método de Hargreaves-Samani superestimou a evapotranspiração em $0,80 \mathrm{~mm} \mathrm{~d}^{-1}$, sendo que a maior superestimativa foi observada para a cidade de Arinos, da ordem 1,23 $\mathrm{mm} \mathrm{d}^{-1}$, que é um erro elevado para uma estimativa diária.

Os valores da raiz quadrada do erro médio variaram de 0,59 a $1,29 \mathrm{~mm} \mathrm{~d}^{-1}$ para o método de PM-FAO56 (dados incompletos), com um valor médio de $0,80 \mathrm{~mm} \mathrm{~d}^{-1}$; para o método de Hargreaves-Samani, a REQM variou de 0,82 a $1,44 \mathrm{~mm} \mathrm{~d}^{-1}$, com um valor médio de $1,12 \mathrm{~mm} \mathrm{~d}^{-1}$.

O EAM apresentou uma variação de 0,47 a $1,04 \mathrm{~mm} \mathrm{~d}^{-1}$ para o método de PM-FAO56 (dados incompletos), com média de $0,64 \mathrm{~mm} \mathrm{~d}^{-1}$. Já o método de Hargreaves-Samani teve um EAM variando de 0,63 a $1,26 \mathrm{~mm} \mathrm{~d}^{-1}$, com a média de $0,96 \mathrm{~mm} \mathrm{~d}^{-1}$. Os valores próximos do viés médio e do erro absoluto médio mostram que o método de Hargreaves-Samani tende a superestimar o método-padrão durante todo o ano. Assim, o erro absoluto médio aproxima-se do viés médio.

Originalmente, o método de Hargreaves-Samani foi desenvolvido para clima semiárido, e como se baseia apenas em dados de temperatura, espera-se que este método superestime a ETo em climas úmidos (SENTELHAS et al., 2010; ADEBOYE et al., .2009; SUBBURAYAN et al., 2011), como observado nas localidades estudadas.

O método de Hargreaves-Samani apresenta resultados aceitáveis, considerando-se que utiliza apenas dados de temperaturas máximas e mínimas, e uma solução seria ajustar essa equação para as localidades estudadas, como realizado por TRAJKOVIC (2007).

GOCIC \& TRAJKOVIC (2010) desenvolveram um software para o cálculo do ETo na ausência de alguns dados climáticos para a região de Davis na Califórnia. Estes autores recomendam o método de Penman-Monteith quando os dados climáticos são limitados.

\section{CONCLUSÕES}

As estimativas de ETo resultantes da utilização de dados limitados, seguindo os procedimentos descritos no boletim 56 da FAO, mostraram-se adequadas na ausência dos dados de umidade relativa. O pior desempenho foi na ausência de dados de radiação solar.

$\mathrm{O}$ método apresentou bons resultados na ausência dos dados de velocidade do vento, sendo que melhores resultados podem ser obtidos se a média mensal da velocidade do vento estiver disponível.

Quando há disponibilidade somente de dados de temperaturas máximas e mínimas do ar, o método de Penman-Monteith, calculado na ausência de dados, apresentou melhores resultados do que o de Hargreaves-Samani, sendo que a única exceção foi na cidade de Monte Azul.

\section{AGRADECIMENTOS}

Os autores agradecem ao CNPq - Conselho Nacional de Desenvolvimento Científico e Tecnológico, pelo apoio ao desenvolvimento deste trabalho, e ao INMET - Instituto Nacional de Meteorologia, pela disponibilização dos dados utilizados. 


\section{REFERÊNCIAS}

ADEBOYE, O.B.; OSUNBITAN; J.A.; ADEKALU K.O.; OKUNADE, D.A. Evaluation of FAO-56 Penman Monteith and temperature based models in estimating reference evapotranspiration using complete and limited data, application to Nigeria. Agricultural Engineering International, v. 11, n. 1291, p. 1-25, 2009.

ALLEN, R. G.; PEREIRA, L. S.; RAES, D.; SMITH, M. Crop e vapotranspiration: Guidelines for computing crop water require ments. Rome: FAO, 1998. 300 p. (FAO - Irrigation and Drainage Paper, 56).

GAVILAN, P.; BERENGENA, J.; ALLEN, R.G. Measuring versus estimating net radiation and soil heat flux: impact on Penman-Monte ith reference ET estimates in semiarid regions. Agricultural Water Management, Amsterdam, n. 89 n. 3, p. 275-286, 2007.

GOCIC, M.; TRAJKOVIC, S. Software for estimating reference eva potrans piration using limited weather data. Computers and Electronics in Agriculture, New York, v .71, n. 2, p 158-162, 2010.

HARGREAVES, G.H.; SAMANI, Z.A., Reference crop evapotrans piration from temperature. Applied Engineering in Agriculture, St Joseph, v.1 n.2, p.96-99, 1985.

JABLOUN, M.; SAHLI, A. Evaluation of FAO-56 methodology for estimating reference evapotranspiration using limite d climatic data: applications to Tunísia. Agricultural Water Management, Amsterdam, n. 95, p. 707-715, 2008.

CAI, J.; LIU, Y.; LEI, T.; PEREIRA, L.S., Estimating reference evapotranspiration with the FAO Penman-Monteith equation using daily weather forecast messages. Agricultural and Forest Meteorology, Amsterdam, v. 145, p. 22-35, 2007.

ORTEGA-FARIAS, S.; IRMAK, S.; CUENCA, R. Special issue on evapotranspiration measure ment and modeling, New York, Irrigation Science, v. 28 n. 1, p. 1-3, 2009.

POPOVA, Z.; KERCHEVA, M.; PEREIRA, L.S. Validation of the FAO methodology for computing ETo with limite data. A pplication to South Bulgaria, Slough, Irrigation and Drainage, v. 55, p. 201215, 2006.

SENTELHAS, P.C., GILLESPIE, T.J., SANTOS, E.A. Evaluation of FAO Penman-Monte ith and alternative methods for estimating reference evapotranspiration with missing data in Southern Ontario, Canadá. Agricultural Water Management, Amsterdam, v. 97, n. 5, p. 635-644, 2010.

STÖCKLE, C.O., KJELGAARD, J., BELLOCHI, G. Evaluation of estimates weather data for calculating Penman-Monte ith reference crop evapotranspiration, Slough, Irrigation Science, n. 23, p. 39-46, 2004.

SUBBURAYAN, S., MURUGAPPAN, A., MOHAN, S. Modified Hargreaves Equation for Estimation of ETo in a Hot and Humid Location in Tamilnadu State, India. International Journal of Engineering Science and Technology, Tehran, v. 3, n .1, 2011.

TRAJKOVIC, S. Hargreaves vers us Penman-Monte ith under humid conditions. Journal of Irrigation and Drainage Engineering, New York, v. 133, n. 1, p. 38-42., 2007.

VEGA, E. C.; JARA, J. C. Estimación de la evapotranspiración de referencia para das zonas (Costa Y Región Andina) del Ecuador. Engenharia Agrícola, Jaboticabal, v. 29, n. 3, set. 2009.

WIDMOSER, P. A disc ussion on and alternative to Penman-Monte ith equation. Agricultural Water Management, Amsterdam, n. 96, p. 711-721, 2009.

WILLMOTT, C. J. Some comments on evaluation of model performance, Bulletin of American Meteorological Society, Boston, v.63, p. 1309-1313, 1982.

XING, Z.; CHOW, L.; MENG, F.R.; REES, H.W.; STEVENS, L.; MONTEITH, J., Validating evapotrans piration equations using Bowen Ratio in New Brunswick. Maritime Canada. Sensors, Lausanne, n. 8. p. 412-428, 2008. 